\title{
Application and Study on Problem-Based in Computer Network Teaching
}

\author{
Zhengqin Xu*, Lei Zhang, Wendong Zhao, Laixian Peng, Aijing Li \\ College of Communication Engineering, Army Engineering University, Nanjing, China

\section{Email address:} \\ 155267201@qq.com (Zhengqin Xu), leizhang@163.com (Lei Zhang),nj_mouse@163.com (Wendong Zhao), \\ penglaixian@sina.com (Laixian Peng), lishan_wh@126.com (Ajing Li) \\ ${ }^{*}$ Corresponding author
}

\section{To cite this article:}

Zhengqin Xu, Lei Zhang, Wendong Zhao, Laixian Peng, Aijing Li. Application and Study on Problem-Based in Computer Network Teaching. Science Discovery. Vol. 6, No. 5, 2018, pp. 386-391. doi: 10.11648/j.sd.20180605.22

Received: September 19, 2018; Accepted: October 15, 2018; Published: October 18, 2018

\begin{abstract}
Problem-Based Teaching is a teaching method based on problems, in which the teacher combs the knowledge of the course and establishes the chain of questions. So students try to find solutions of them in the learning process. Computer network course is the core course of computer specialty. It involves many knowledge points which is scattered and covering a wide range, so that it is abstract and difficult to understand. Students feel it is difficult to learning because there is no direct link between various protocols. This paper analyzes the existing problems in computer network teaching, puts forward the application of "Problem-Based" interactive teaching mode in computer network course, puts forward that creating problem situation on the basis of theoretical analysis and practice is an effective teaching strategy, and defines the design principles of effective problems. Practice has proved that the application of Problem-Based Teaching method in computer network course can stimulate students' interest in learning, enhance students' self-learning awareness and cultivate students' self-learning ability. This paper takes the "identification protocol" as an example and expounds in detail how to cultivate the students' ability to apply computer network knowledge by creating a series of question chains in computer network teaching.
\end{abstract}

Keywords: Problem-Based Learning, Chain of Problems, Authentication Protocol

\section{问题驱动式在计算机网络教学中的应用与研究}

\author{
徐正芹“，张否，赵文栋，彭来献，李艾静
}

通信工程学院, 陆军工程大学, 南京, 中国

\section{邮箱}

155267201@qq.com (徐正芹), leizhang@163.com（张磊）, nj_mouse@163.com（赵文栋）, penglaixian@sina.com（彭来献）, lishan_wh@126.com (李艾静)

\footnotetext{
摘要: 问题驱动教学法是以问题为牵引, 由教员梳理课程中的知识点并建立问题链, 使学员在学习过程中始终围绕问 题, 不断寻求解决方法的一种教学方法。计算机网络课程是计算机专业的核心课程, 它涉及知识点多、散, 涵盖面广, 内容抽象难以理解, 各协议层次间没有直接联系, 不易被学生掌握。文章分析了计算机网络教学中存在的问题, 提出 了“问题驱动式”的互动教学模式应用于计算机网络课程, 提出在理论分析和实践的基础上创设问题情境是有效的教学 策略, 并界定有效问题的设计原则。实践证明, 在计算机网络课程中应用问题驱动式教学法能激发学员的学习兴趣, 增强学员的自主学习意识, 培养学员的自主学习能力。文章通过“鉴别协议”的实例教学, 详细阐述在计算机网络教学 中如何通过创设一系列问题链培养学员 “计算机网络知识”的学以致用的能力。
} 
关键词：问题驱动，问题链，鉴别

\section{1. 引言}

计算机网络课程是计算机专业的主干课程, 是教授 计算机网络理论知识和解决网络实际问题的一门重要的 专业基础课, 在网络工程专业人才培养中起着基础性和 先导性的作用。但是在教学过程中发现存在学员学习积 极性不高, 考试通过率低的等诸多问题。

经过分析, 我们发现造成这些问题的原因主要有以 下几点:

（1）知识面涵盖范围广。计算机网络是一门与通信 技术等学科有交叉的课程, 知识点多且杂, 内容涉及到 通信、数据结构、网络原理及众多的网络协议。

（2）网络协议抽象难懂。网络中的协议内容复杂, 对于初学者来说, 看不见摸不着, 十分抽象。很多学员 在学习协议时会被复杂的协议吓住, 不敢也不愿继续深 层次学习。

（3）各章节内容之间没有逻辑关系。不像其它课程, 通常前一章是为后一章做铺设, 计算机网络的各章基本 上都是独立的, 是并列的关系, 通常可以改变学习的先 后顺序。比如有的教材按照网络结构体系自顶向下介绍, 有的教材则按照自底向上的顺序介绍。对于初学者来说, 通常一类协议还没理解, 又开始了另一类协议, 各种协 议杂乱无章, 就像一团乱麻, 使学员感到一头雾水, 毫 无头绪[1]。

基于计算机网络课程所有的特点, 在教学中, 教员 应该采用合理的教学方法, 帮学员建立整个网络体系结 构。但是目前大多数院校教员仍然采用传统的授课方式。 传统的课程的实施是教员根据事先设计好的教案授 课, 主要采取“教员讲, 学员听”的被动、机械接受知识 的传统教学模式。这种做法在一定程度上忽视了学生的 积极性, 缺乏对教学信息的适时检查和反馈, 不知道学 生是否理解了复杂而抽象的网络知识, 不能实现对教学 过程的及时调节和最佳控制; 教员的讲授只看重现有的 网络协议及其应用, 作业和练习的目的也都是为了应试, 对于协议的来龙去脉, 不做任何引导和解释。

如果按照这种模式，教员与学员之间交流机会少, 学员就会感觉课程枯燥无味, 自然他们的学习兴趣就会 不高, 学习效果不理想。

为了解决这个问题, 本文提出将问题驱动式教学应 用于计算机网络的教学中。在教学过程用恰如其分的问 题引导每次课程要学习的内容, 通过精心设计问题, 以 问题为导向, 使学员置身于思考问题、解决问题的动态 学习过程当中, 从而使学员产生浓厚的兴趣和强烈的解 答欲望, 牢牢吸引他们的注意力, 充分发挥他们学习的 主观能动性, 从而优化课堂教学, 提高教学质量, 达成教 学效果。

\section{2. 问题驱动式教学模式}

\section{1. 问题驱动式教学的概念}

法伏尔泰说得好 “令人讨厌的艺术就是把什么都说了 出来”。问题驱动式教学法就是以“问题”为载体, 在课堂 教学中通过设计一系列的“问题链”, 以此来引导学生学 习、合作研究。使学生在解决问题的过程中得到进步, 从而提高了学生综合的素质[2]。

问题驱动式教学法的渊源可以追溯到19世纪后期美 国实用主义教育家杜威提出的“情境、问题、假设、推理 和验证”的教学模式, 其强调“从做中学”的思想对整个教 育界产生了很大影响[3]。1960年, Barrows和Tamblyn在 医学教育领域提出了问题驱动式学习(Problem-Based Learning, PBL)教学法, 以克服刚毕业的医科学生虽有 大量专业知识却缺乏临床应用能力的缺点 [4]。1969年, 加拿大麦克马斯特大学首先在医学领域建立了以 PBL为 主的教育策略, 之后逐步推广到商学、教育学、建筑、 法律、工程等学科的教学活动中, 对于培养学员自主创 新和解决问题的能力具有明显效果。美国的哈内曼大学 在公共健康学院、普渡大学在牙齿卫生学科实行了这种 教学法 [5]。现阶段问题驱动式教学法在西方国家各教育 领域有了更深入发展, 他们的课堂教学更注重启发诱导, 自主探究, 重视学员创新思维的培养。在这种教育下, 学员也表现出了较强的创新意识和研发能力。

中国著名教育家陶行知说过: “创造始于问题, 有了 问题才会思考, 有了思考, 才有解决问题的方法, 才有 找到独立思路的可能”[6]。问题驱动式教学正是以探索问 题来引导和维持学生的学习兴趣和动机, 使学生的学习 不单是知识由外到内的转移和传递, 更应该是学生主动 建构自己知识经验的过程, 通过新经验和原有知识经验 的相互作用, 充实和丰富自身的知识、能力[7-9]。

\section{2. “问题驱动式”教学法的优势}

“问题驱动式”教学法的目的不仅能够增长学员的知 识储备, 更能使学生参与到分析问题、解决问题的过程 中, 培养学员的思维能力和创新能力, 增强学员的问题 意识。“问题意识”是指推动学员自己思考的动力, 是促 使学员不断质疑、探索的动力。利用 “问题驱动式”教学 模式, 使课堂活页, 促使学员积极参与分析、探究、解 决问题。问题吸引学员注意力增强其注意力的指向性与 选择性, 刺激学员思考, 避免思维定势, 提高创新思维。

\section{3. 有效问题的设计原则}

问题驱动式教学, 如何设计一系列有效问题, 是一 个关键点。学员学习的愿望总是在一定的问题情境下产 生的, 好的问题总是能激起学员强烈的学习欲望。

如何设计有效问题呢, 在教学过程中问题的设计原 则应遵循以下几点[10]: 
(1) 问题的设计应该有针对性, 即针对一定的教学 目标提出来的, 问题要有意义, 有明确的指向性, 能反 映学员当前学习的内容。

例如, 在学习“配置路由器的路由选择协议”一节中, 由于涉及到IP地址的划分、路由协议的配置等众多知识 点, 许多学员不能正确的进行子网划分, 这时可以引导 学员回忆子网划分的概念, 以及不同子网之间如何进行
分组通信。还有一些学员经常会忽略了配置主机默认网 关而导致无法Ping通其它设备, 此时可以启发学生默认 网关的作用是什么? 主机为什么需要默认网关等。当学 生完成协议配置并测试成功后, 可以进一步启发他们思 考分组在PC0 到达PC1的过程中其IP地址与 MAC地址分别 是怎么变化的? 并让其在PacketTracer中实践模拟分析。

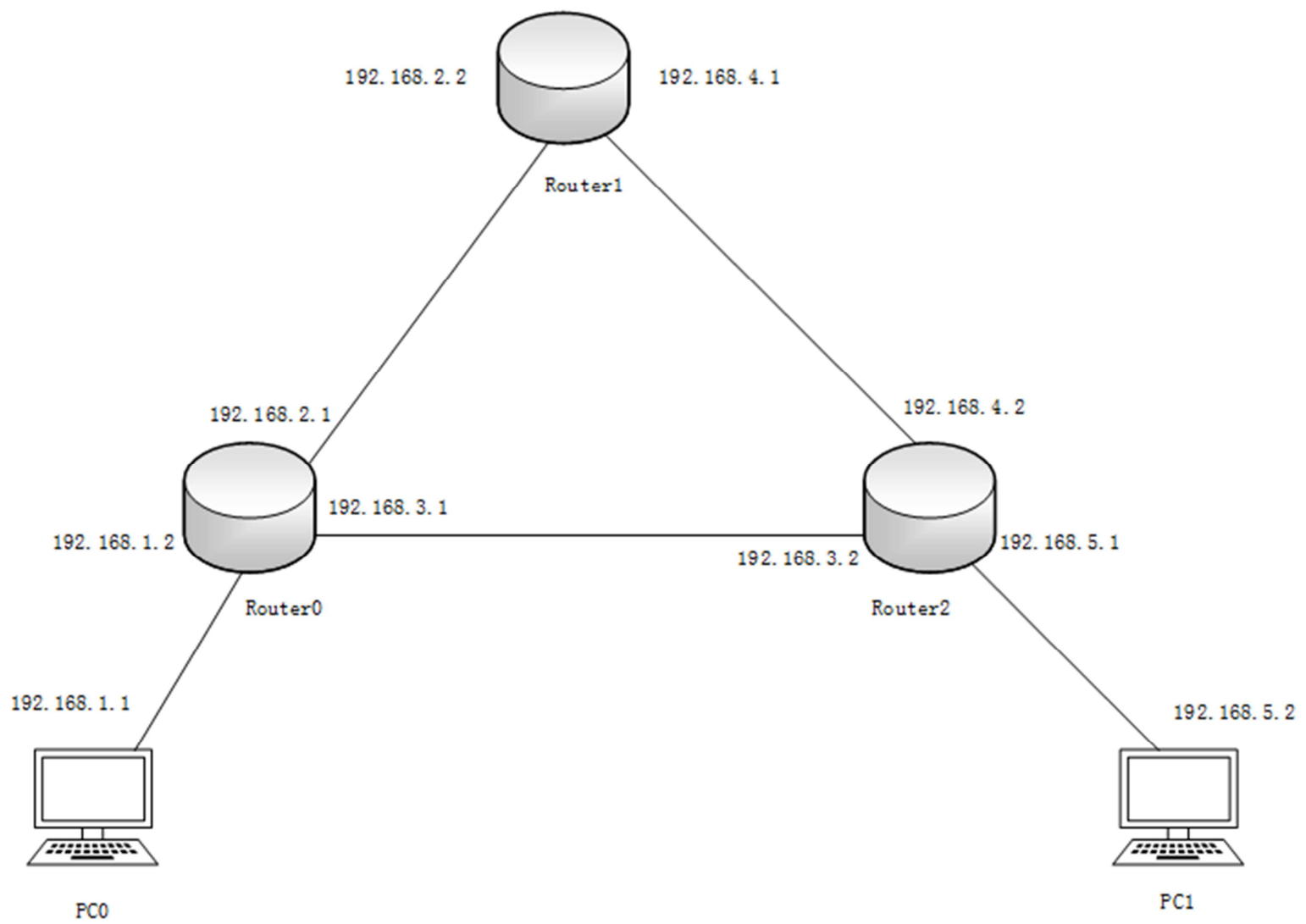

图1 路由协议配置拓扑结构。

(2) 问题的设计应该立足于学员已有的经验和已学 过的知识, 问题的难度要合适, 在教员的引导下, 使学 员独立自主地思考和解决问题。

课堂问题的设计以激发学生探究兴趣为原则, 能激 发学生的学习兴趣, 使学生产生强烈的探索欲望和求知 欲望。以本人教授的《计算机网络》课程为例, 在教学 过程中往往会联系生活中的网络应用知识, 在恰当的时 候提出和知识点相关的问题, 并根据学员已有知识加以 引导, 这样做能够激发学生的兴趣, 钻研并解决问题, 进而产生的强烈的成就感和自豪感。而这种感情又会进 一步激发学生去再发现、再探索、再创造。

(3) 课堂问题的设计与解决应该具有渐进性。课 堂上提出的问题尽量不要一步到位, 应该具有递进性和 层次性。教员在设计问题时要符合学生的思维习惯, 针 对学生的具体情况由浅入深地设计一系列具有逻辑性的 问题, 并且要注意合理安排问题的次序, 特别是对内容 的重点、难点, 通过精心设计, 依次铺设问题, 引领学 员“拾阶而上”, 积极主动地获取知识。比如讲“可靠传输 协议”一节, 教员可以提问 “如果在完全可靠的信道, 数
据如何传输的? ”, 接着提出“若有比特差错的信道, 数 据应该如何可靠传输？”，最后提出“具有比特差错的丢 包信道, 数据应如何做到可靠传输? ”。随着问题的层层 递进, 最终得到一个无错的可靠的数据传输协议。

(4) 问题的设计要有发展性: 提出的问题要给学生 提供探究和发现的体验, 问题能入手, 又有挑战性, 研 究的间较大, 有助于培养学员的创造思维。

\section{4. 问题驱动式教学实施步骤}

在问题驱动教学法实施的过程中, 可以分为以下几 个步骤[11-12]: (1)创设情境, 提出问题; (2)剖析、探索、 解决问题; (3)归纳成果、总结与反思。

在实际教学中, 我们可以根据以上步骤组织问题驱 动式教学。

第一个环节是创设情境, 针对具体教学内容, 在理 论分析和实践的基础上创建真实的应用环境, 激发学生 兴趣。

第二个环节是教员引导学员解决问题, 这一环节可 以采用学员自主思考或互相讨论的方式。在这个环节, 
应该凸显学员的主体地位, 帮助学员实现自我激励和自 我监控, 使课堂教学过程建立在多边互动基础上, 进而 使课堂教学形成高潮, 同时也有助于教员了解学员学习 情况和个人特点。

第三个环节是由教员或学员在已经解决的问题基础 上归纳总结, 并提出新的问题。

\section{3. 问题驱动式教学法的实例}

为了更好的说明问题驱动式教学法在计算机网络中 的应用, 我们以“鉴别协议”一节的教学为例, 详细阐述 如何在教学过程中创设情境、提出有效问题, 最终如何 引导学员解决问题。

\section{1. 第一环节: 设立情境}

本次课以美军的一次名为“联合勇士”的演习为引入, 海军指挥系统被一名空军上尉成功入侵, 并取得舰队控 制权, 引出课题——系统服务器对空军上尉的身份鉴别 出现了问题: 如何设计鉴别协议?

情境设计: “在计算机网络无处不在的今天, 每天都 会登录QQ、微信系统、校园网, 服务器如何鉴别客户端 的登陆的呢？请设计一个校园网登录的单向鉴别协议。” 设计三个角色: 客户端Alice通过网络向服务器Bob证实 自己的身份。Sam是一个入侵者。

此情境设立以学员熟悉的军事案例和实际生活中遇 到的情况为引入, 设置问题, 可有效地提起学员探究的 心理, 点燃学员思维的火花, 从而形成问题意识, 激发 学员解决问题的潜能和学习兴趣。

\section{2. 第二环节: 解决问题}

本环节在协议设计过程中以一系列“问题链”作为整 堂课的纽带, 问题的设计层层递进、循序渐进, 直到最 终解决问题。

问题1:

请同学们根据平时的经验, 设计出一个身份鉴别一 一协议1.0。

协议1.0 Alice明文发送“我是Alice”和口令给Bob。

教员问题: 1.0协议能否被Sam窃听? 如果能被窃听, 应该怎么改进?

经过讨论后, 得出结论由于没有任何验证机制和保 密措施, 很容易被Sam窃听, 从而冒充Alice。

问题 2:

教员设问：解决协议 1 的漏洞—如何不被窃听?

学员经过讨论提出: 可以利用加密口令, 设计出协议

2.0

协议2.0 Alice发送我“我是Alice”和加密口令给Bob。

教员问题: 如何加密?

学员结论: (讨论后) 利用上节课学过的对称密钥 加密口令。

教员问题: Sam无法知道口令确切内容, 是否就不 能冒充Alice?

用问题激发学员的兴趣和探索欲望, 然后引出即使 不知道口令确切内容, 只需要记录口令的加密版本, 回 放给Bob，即可冒充Alice。
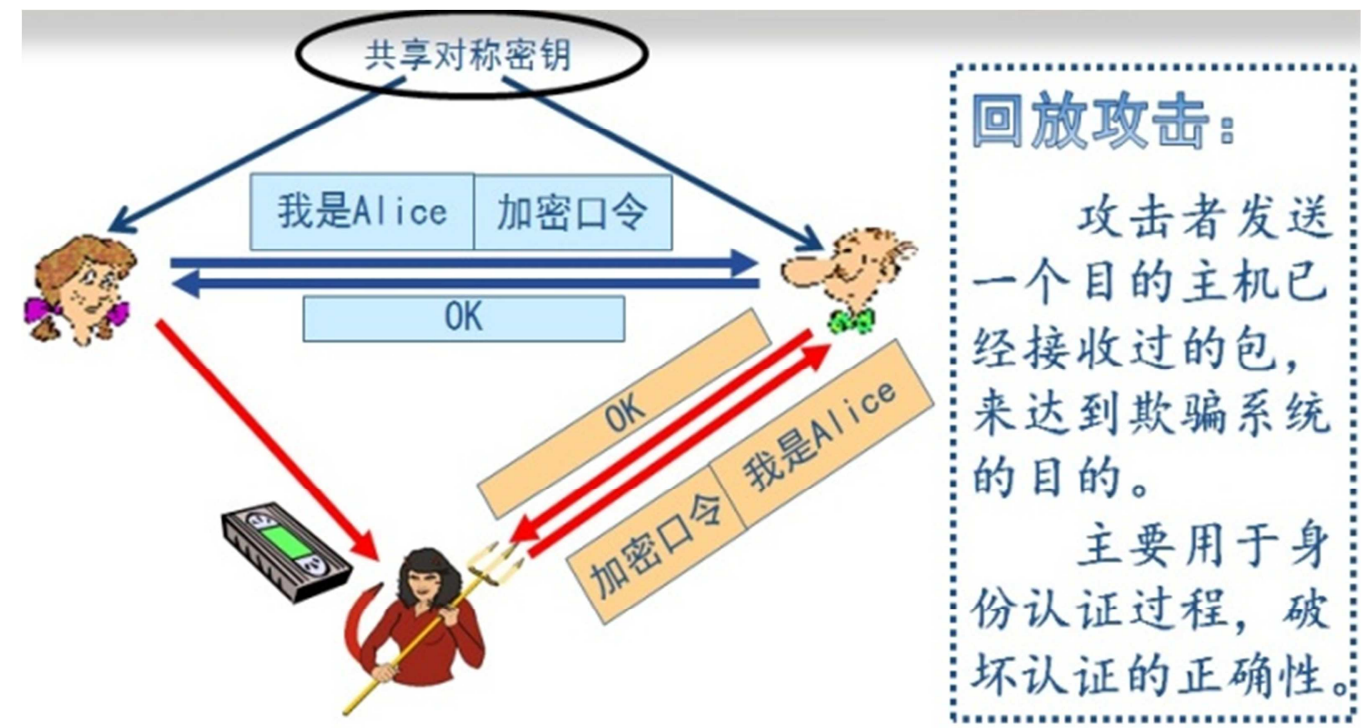

图2 协议2.0。

问题3:

教员问题: 启发学员, 协议 2.0 中 Sam之所以能冒充 Alice的原因是什么?

是由于Bob不知道Alice是否还在连接的另一端, 是 否还“活着”。
教员提出问题: 这个问题在计算机网络中是否遇到 过?

经过学员讨论, 得出这个问题跟 TCP三次握手协议 有异曲同工之处, 可以采取同样的策略解决。 


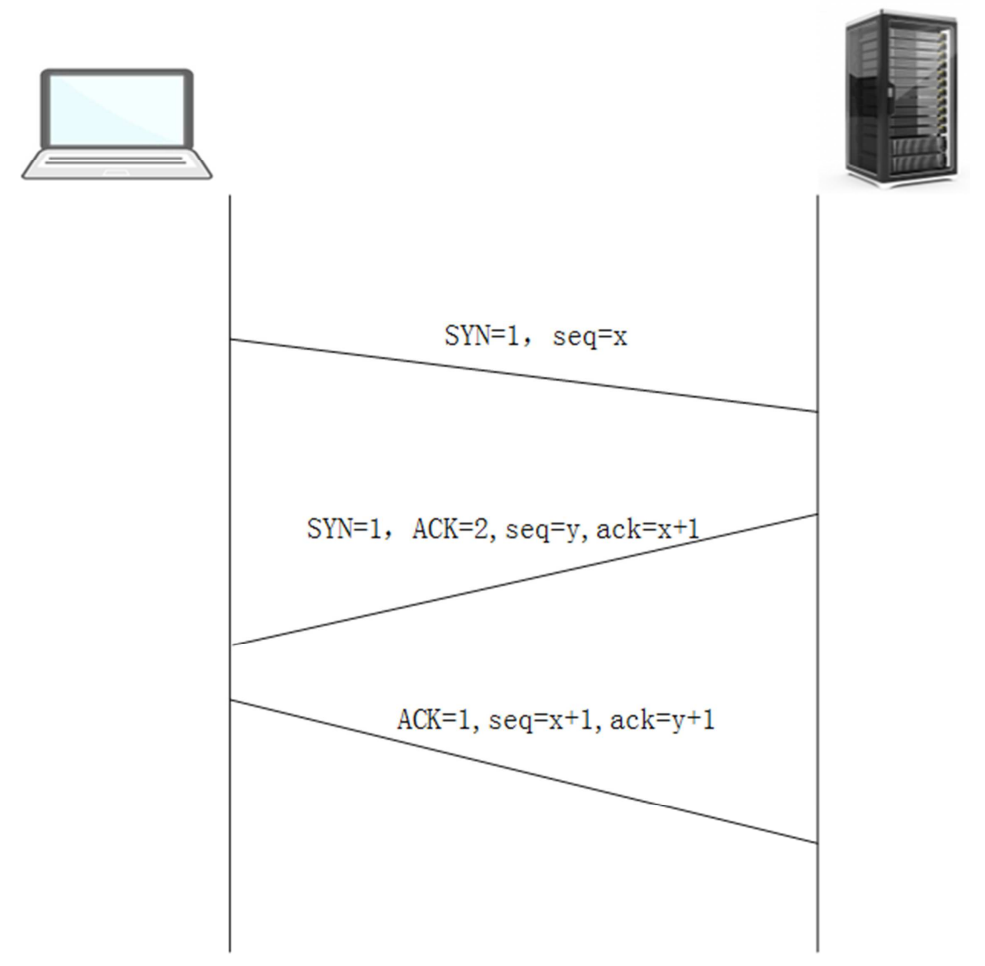

图3 TCP三次握手协议示意图

最后总结: 利用共享对称密钥 $K a-b$ 和不重数 $R$ 可以成 功设计此协议。

协议3.0, 如图3所示, 具体协议描述如下:

(1) Alice 向bob发送报文“我是Alice”

(2) Bob选择一个不重数R, 发送给Alice
（3）Alice使用她与Bob共享的对称密钥Ka-b加密不 重数,并将加密的不重数Ka-b (R)发给Bob

(4) Bob解密接收的报文, 若解密得到的 $R^{\prime}=R$, 则 可确定Alice的身份。

\section{鉴别协议3.0}

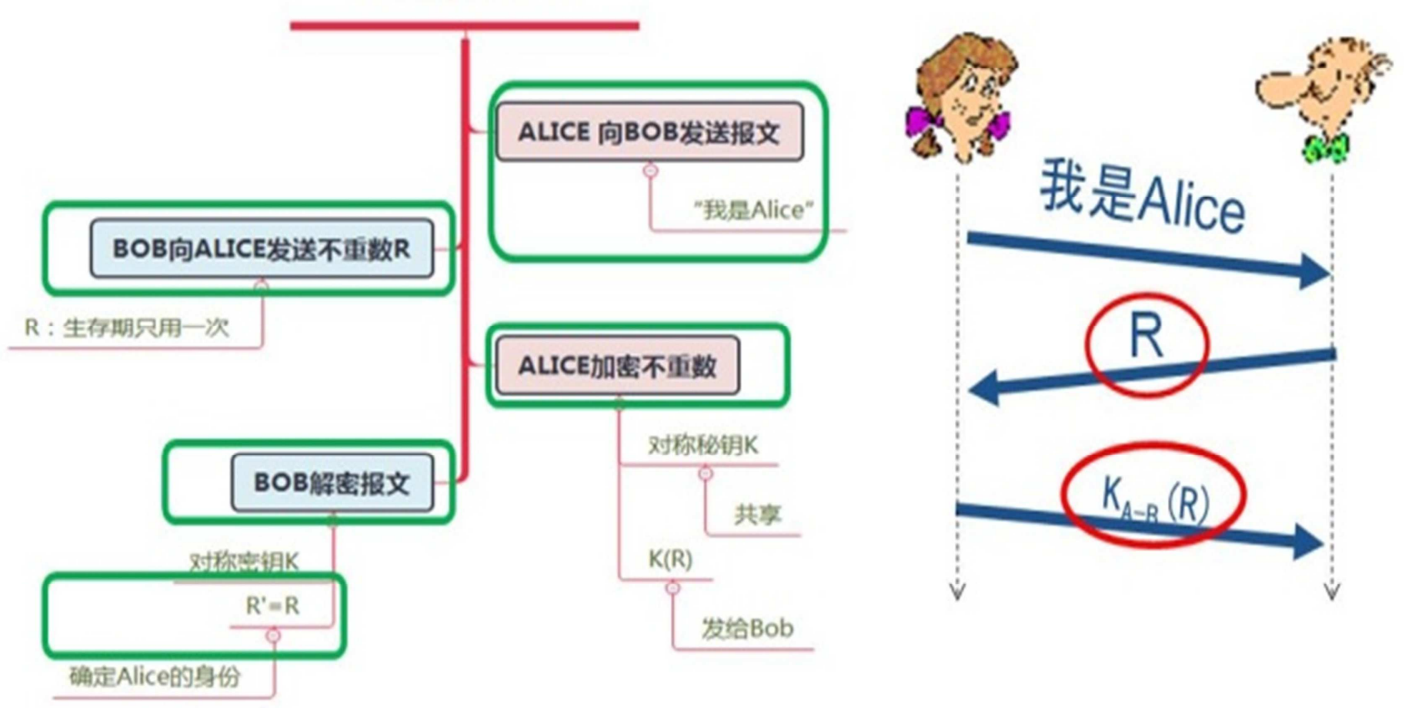

图4 协议3.0。

解决问题“如何设计一个校园网登录的鉴别协议?”

教员设问：设计此协议的关键是什么?

学员讨论答: 选择合适的对称密钥, 并利用协议 3.0

提示：客户端注册后，服务器端会在数据库中保存

用户名和口令。
学员讨论后, 得出结论: 可选口令作为共享对称密 钥。

协议设计如图4所示: 


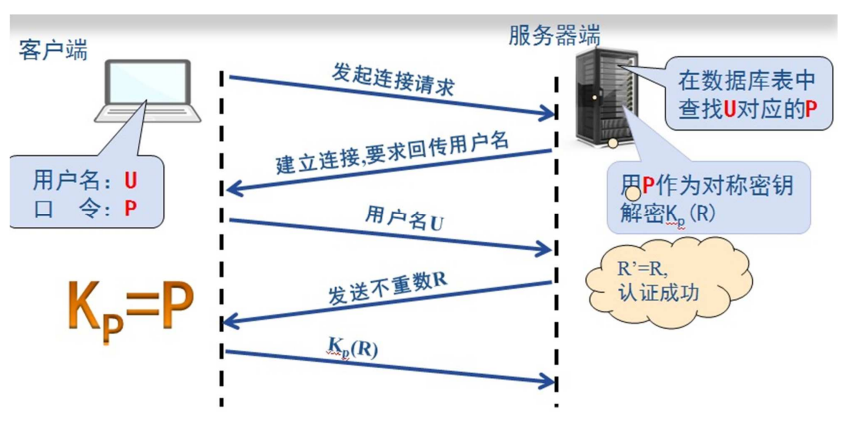

图5 校园网登录鉴别协议。

在此环节, 所设置的问题像阶梯一样由低到高、由 易到难, 逐步递进, 引导学员的思维步步深化, 逐步设 计了协议 1.0 直到协议 3.0 , 引导学员在设计过程中发现问 题、探究问题和解决问题, 从而自主构建知识框架, 并 最终解决了课堂开始时所提出的问题。

\section{3. 第三环节: 总结评价, 提出新问题}

总结、评价：引导学员思考“单向鉴别协议为什么能 用共享对称密钥和不重数设计? ”“单向鉴别协议, 能利 用共享对称密钥的前提是只需要客户端向服务器端证明 身份, 而服务器端不需要向客户端证明身份, 即基于客 户端对服务器端的绝对信任, 所以客户端可以将自己的 共享密钥发给服务器端。”

提出问题: “对于双向鉴别协议, 比如两个军用电台 通信前, 身份鉴别协议的设计是否还能继续使用共享对 称密钥和不重数来设计? 如果能会带来哪些问题? 如果 不能用共享对称密钥, 可以采用哪种方式呢? ”

通过教员一系列问题的引导, 经过学员的讨论探索, 最终达到掌握协议的设计方法, 及如何发现和处理协议 设计中的漏洞, 并最终能自行设计一个可靠的鉴别协议。

\section{4. 结论}

问题驱动教学法, 通过合理设置启发式、探索式和 温故式的问题, 激发学员学习热情, 通过课堂讨论、提 问、建立小组等形式加强师生互动。

在实践过程中应该注意以下几个方面:

(1) 科学的制定问题。问题如何设置关乎到教学效 果的好坏, 所以应该在设置问题前仔细分析教学目标和 内容, 设置的问题最好能起到抛砖引玉的效果, 教员可 通过旁敲侧击、点拨诱导, 使学员对知识达到融会贯通, 心领神会, 最终达到解决问题的目的。

(2) 找准教员的角色定位, 在过程中时刻把握主线, 做课堂的导演。教员将问题抛给学员后, 不要急于给出 答案, 可以围绕所提出的中心问题, 布阵设疑, 搭桥铺 路, 创设问题情境, 让思维的火花从学员自己的脑海中 迸发出来, 这对学员的悟性和创新思维极为有利。

(3) 培养学员的团结合作和自主思考的精神。以学 员自主探讨为主, 同时基于教员自己所掌握的经验、知
识、对问题以及学员知识结构的了解, 对学员进行合理 分工, 对学员的探索活动给予积极有效的指导, 通过学 员和学员之间、小组和小组之间的交流探讨, 既可以增 强学员的探索和创新能力、学习科学研究的方法、培养 综合运用知识的能力, 还可以培养学员的团队合作精神 和竞争意识, 促进学员的全面发展。

在运用问题驱动教学法之前, 学员学习计算机网络 知识往往感到内容复杂网络协议抽象又难以理解, 导致 学员不愿意做深入的学习和思考。我们在网络中的各种 协议、各层服务等教学问题中运用问题驱动式教学法之 后, 学员对学习的内容能很快掌握, 并能融合贯通, 举 一反三, 真正做到知其然, 也知其所以然。学员在不知 不觉中养成了思考的习惯, 培养了创新的思维。

\section{参考文献}

[1] 陈鸣, 胡谷雨, 周雷等.计算机网络课程教学的思考与创 新[J].高等教育研究学报, 2008 (2)。

[2] Mohammad Imran, Problem-based learning versus lecturebased learning in pharmacology in a junior doctor teaching program: a crossover study from northern India [J], International Journal of Research in Medical Sciences, 2015, Nov; 3 (11): 3296-3299.

[3] Agus Salim, The Problem Based Learning Model To Improve The Students' Critical Thinking Ability [3], Journal of Research \& Method in Education, 2018, Mar (2): 36-40

[4] Ilhan KARATAS, The Effect of Learning Environments Based on Problem Solving on Students' Achievements of Problem Solving [J], International Electronic Journal of Elementary Education, 2013, 5 (3): 249-268.

[5] Melanie LaForce, Problem-Based Learning (PBL) and Student Interest in STEM Careers: The Roles of Motivation and Ability Beliefs, Education Science, 2017, 7: 4-6.

[6] 范福生, 问题驱动教学法在物理教学中的应用 $[\mathrm{J}]$. 教学与 管理, 2012，6(18): 141-143。

[7] 冯迪, 杨明伟, 周震。信息光学及光信息处理“问题驱动” 教学方式探索 $[\mathrm{J}]$. 教育教学论坛, 2012, 10 (30): 40一4。

[8] 张霞, 建构主义教学观下的支架式教学实证研究 [J]. 教学 研究, 2011, (11): 150-153。

[9] 张明芳, 试论运用建构主义支架式教学模式实施口译教学 [J]. 江西教育学院学报, 2013, (6): 121-124。

[10] 曲涛. 支架式教学在幼儿园集体教学中的应用探究 $[\mathrm{J}]$. 学 园, 2013，(32)：162-164。

[11] 高艳. 基于建构主义学习理论的支架式教学模式探讨 [J]. 当代教育科学, 2012, (19)：62-65。

[12] 王喜耘. 幼儿语言教育存在的问题与对策 $[\mathrm{J}]$. 基础教育, 2014, (3): 47-51。 